\title{
ORIGINAL RESEARCH \\ Balancing Radiation Dose and Image Quality: Clinical Applications of Neck Volume CT
}

\author{
M.T. Russell \\ J.R. Fink \\ F. Rebeles \\ K. Kanal \\ M. Ramos \\ Y. Anzai
}

BACKGROUND AND PURPOSE: The advancement of multidetector CT technology has resulted in improved image quality as well as an increase in ionizing radiation dose to patient. The purpose of this study was to assess radiation dose and overall image quality of CT examination of the neck between fixed-tube current and automatic tube current modulation (ATCM) at 2 separate noise index levels.

MATERIALS AND METHODS: A total of 84 patients underwent neck CT with use of a 64-section multidetector row CT (MDCT) scanner. Patients were divided into 3 groups: fixed-tube current $(n=28)$, ATCM with a noise index of $11.4(n=28)$, and ATCM with a noise index of $20.2(n=28)$. All other scan parameters remained constant. Scan coverage length and transclavicular distance were measured. Two radiologists blinded to the scanning parameters assessed overall image quality, noise level, and streak artifacts using a 5-point grading scale. The radiation dose in dose-length product (DLP) and CT dose index (CTDI) was recorded.

RESULTS: Compared with a fixed-tube current technique, ATCM with a noise index of 11.4 reduced CTDI by $20 \%\left(P<.01 \times 10^{-6}\right)$, and ATCM with a noise index of 20.2 reduced CTDI by $34 \%(P<.01$ $\left.\times 10^{-12}\right)$. Although the difference in image quality between the fixed-tube current technique and the noise index reached statistical significance $(P<.05)$, the magnitude of the difference was small, with average scores of $3.79( \pm 0.59)$ and $3.57( \pm 0.53)$, respectively.

CONCLUSION: Compared with the fixed-tube current technique, ATCM resulted in significant reduction of radiation dose without substantially reducing the image quality of the CT of the neck. Judicious monitoring of radiation dose to patients has to be balanced with diagnostic image quality.
A lthough CT accounts for $11 \%$ of $\mathrm{x}$-ray-based imaging in the United States, it is responsible for more than $67 \%$ of the radiation dose associated with all medical imaging techniques. ${ }^{1}$ As newer and faster scanners are developed, individual patient doses are increasing because of volume scanning, thinner sections, overlapping scans, and increasing scan coverage. ${ }^{2}$ As application of CT is becoming more widespread, it is important to remember the concepts of radiation protection and radiation dosimetry to keep the collective dose of radiation as low as reasonably achievable (ALARA). ${ }^{3}$ At the same time, image quality must remain high enough to preserve diagnostic use.

Radiation dose is determined by several scanning parameters such as beam energy (tube voltage), tube current-time product (mAs), section thickness, number of sections, and pitch, ${ }^{4}$ as well as body habitus of the patient. The automatictube current modulation (ATCM) technique enables automatic adjustment of the tube current in various planes ( $\mathrm{x}-\mathrm{y}$ or z) based on the size and attenuation of the body area scanned to achieve constant image quality. ${ }^{4,5}$ The fixed-tube current technique uses the same single-tube current throughout a continuous helical acquisition, whereas $\mathrm{Z}$-axis modulation changes the tube current from 1 section position to the next. Angular modulation changes the tube current within the section in the $\mathrm{x}-\mathrm{y}$ plane. A combination of the $\mathrm{z}$-axis and angular

Received May 18, 2007; accepted after revision October 11

From the Department of Radiology, University of Washington, Seattle, Wash.

Paper presented previously at: Annual Meeting of the American Society of Head and Neck Radiology, September 28, 2006; Chandler, Ariz. This presentation received the 2006 ASHNR Resident-in-Training Award.

Please address correspondence to Yoshimi Anzai, MD, MPH, Department of Radiology, University of Washington, 1959 NE Pacific St, Seattle, WA 98195-7115; e-mail: anzai@ u.washington.edu

DOI 10.3174/ajnr.A0891 modulations further reduces radiation exposure while achieving a desired constant noise level.

With the ATCM technique, tube current can be automatically reduced while scanning regions of lower attenuation or automatically increasing for areas of higher attenuation while maintaining an acceptable level of image noise. A low noise index selected means less noise at the cost of higher radiation dose, and visa versa. For neck CT imaging with use of ATCM, $\mathrm{z}$-axis modulation adjusts the tube current along the $\mathrm{z}$-axis, which decreases the tube current while scanning through the neck and increases the tube current through the skull base and thoracic inlet. With angular modulation, the tube current increases in the lateral rather than in the anteroposterior/direction at the shoulder.

Previous studies have shown that use of ATCM optimizes radiation dose without compromising image quality in the chest, abdomen, and pelvis. ${ }^{6-11}$ To our knowledge, there is no published study evaluating its use in imaging of the head and neck, and no publication addresses radiation dose and image quality with 64-section multidetector row CT (MDCT). Thus, the purposes of this study were to assess whether the ATCM technique reduces radiation dose compared with the fixedtube current technique without compromising image quality in a 64-section MDCT of the neck.

\section{Materials and Methods}

The Human Research Committee Review Board has approved our study for retrospective review of radiation dose and image quality of contrast-enhanced CT examinations of the neck, with waiver of informed consent.

\section{Imaging Parameters}

We obtained CT examinations of the neck using 64-section MDCT scanners (LightSpeed; GE Healthcare, Milwaukee, Wis) at the Uni- 
versity of Washington with either fixed $\mathrm{mA}$ or ATCM techniques using 2 predetermined noise index values: 11.4 and 20.2. The noise index value is specified by the vendor as "approximately equal to standard deviation in the central region of the image when a uniform phantom is scanned and reconstructed."

The other scanning parameters remained constant for each patient, regardless of the technique. These scanning parameters were $140 \mathrm{kVp}$, pitch 0.987 , gantry rotation time 0.5 second, beam collimation $40 \mathrm{~mm}$, FOV $20 \mathrm{~cm}, 2.5-\mathrm{mm}$ reconstructed section thickness, and a standard reconstruction algorithm. In addition to these parameters, fixed $\mathrm{mA}$ values between 400 and $650 \mathrm{~mA}$ and an auto $\mathrm{mA}$ range of 100 to $750 \mathrm{~mA}$ with a noise index of 11.4 and 20.2 were used. Intravenous contrast material (100-150 mL Optiray; Mallinkrodt, Hazelwood, Mo) was injected through an antecubital vein, and scanning was initiated after a 100-second delay.

\section{Radiation Dose}

Radiation "exposure" is usually a measured quantity, whereas the absorbed radiation "dose" is typically a value calculated from the exposure and from estimates of energy absorbed per body mass unit. The fundamental radiation dose parameter in CT is the CT dose index (CTDI). The volume CTDI $\left(\mathrm{CTDI}_{\mathrm{vol}}\right)$, a derivative of the CTDI (mGy), can be used to express the average dose delivered to the scan volume for a specific examination. CTDI $_{\text {vol }}$ is considered more useful in comparing radiation dose with critical organs such as the thyroid and lens for CT examination of the neck. Dose-length product (DLP $[\mathrm{mGy}-\mathrm{cm}])$, on the other hand, represents the integrated dose for the scan series. DLP will increase with an increase in total scan length or with variables such as tube current or pitch. DLP reflects most closely the radiation dose for a specific CT examination because its numeric value is affected by variance in patient anatomy such as the patient's height. ${ }^{12}$ In this study, we recorded both CTDI $_{\text {vol }}$ and DLP for each patient.

\section{Subject Size and Scan Length}

Although the size and height of the subject influence radiation dose (DLP), body weight or shoulder width was not measured routinely for patients who underwent CT of the neck. Because the shoulder width seems to be a reasonable surrogate of patients' body size, we measured the transclavicular length (measurement taken from the sternal notch, paralleling the clavicle, extending out to the skin marking just below the acromioclavicular joint) on the scout view. In addition, we also measured the total scan length of the neck CT (from the skull base to the superior part of the mediastinum) using the table positions by subtracting the table position of the lowest section from the highest section position and recorded the length in centimeters.

\section{Subjects}

All neck CT examinations performed between November 1, 2005, and January 31, 2006, with use of 64-section MDCT scanners (LightSpeed; GE Healthcare) at our institution were reviewed by the principal investigator. Among them, the following neck CT examinations were excluded from the analysis: neck CT combined with other parts of the body such as the brain, chest, abdomen, or pelvis; neck CT with special protocol other than routine neck examination, such as CT of the larynx that included breathholding and straw blowing with separate acquisitions; and neck $\mathrm{CT}$ performed without intravenous contrast. Studies with fixed mA (400-650 mA) or ATCM with a noise index of 20.2 or 11.4 were selected for the analysis.

Using the above criteria, we performed only 28 studies with a noise index of 20.2, which determined the number of subjects for each technique. We consecutively reviewed cases with fixed $\mathrm{mA}$ and noise index 11.4 techniques from the same study period, then eliminating patients with transclavicular distances of more than $24 \mathrm{~cm}$ or less than $17 \mathrm{~cm}$ as well as cases with scan lengths of more than $400 \mathrm{~cm}$ or less than $200 \mathrm{~cm}$. One patient with noise index $11.4 \mathrm{did}$ not have a radiation dose record for unknown reasons; thus, this patient was eliminated from the study. This resulted in 28 patients with fixed $\mathrm{mA}$ and 28 patients with noise index 11.4 techniques.

There were 12 female and 16 male subjects with a mean age of 52 years (range, 23-81 years) for a noise index of 20.2; 8 female and 20 male subjects with a mean age of 49 years (range, $20-82$ years) for a noise index of 11.4, and 7 female and 21 male subjects with a mean age of 49 years (range, 20-71 years) for fixed $\mathrm{mA}$.

\section{Image Quality}

Two board certified neuroradiologists (J.R.F. and F.R.) blinded to the imaging technique independently reviewed the $2.5-\mathrm{mm}$ reconstructed axial images on PACS. We facilitated the blinding by removing the scanning parameters from the PACS screen. The readers independently graded the studies for overall image quality, noise (mottle, graininess), and streak artifacts using a 5-point grading scale. Overall image quality was graded on the basis of diagnostic acceptability as follows: score of 1 , nondiagnostic study; 2, poor or suboptimal study; 3 , acceptable; 4, good; and 5, excellent studies. Subjective noise on the image was graded as a score of 1 , not acceptable noise level; 2 , definitely noisy images; 3 , slightly noisy but acceptable; 4 , minimal noise not affecting diagnostic quality; and 5, no perceivable noise. Streak artifacts, particularly through the posterior fossa and thoracic inlet, were addressed as follows: score of 1 , severe artifact; 2 , moderate artifact degrading diagnostic capability; 3, slight artifact without interfering with diagnostic capability; 4, minimal artifact; and 5, no perceivable artifact. Window width and levels were adjustable on the PACS workstation by the interpreting radiologists as needed.

\section{Statistical Analysis}

The unpaired $t$ test (Excel 2004, version 11.2.5; Microsoft, Redmond, Wash) was used to compare average transclavicular distance, scan length, DLP, and CTDI ${ }_{\text {vol }}$ with 3 different imaging techniques. The Wilcoxon Mann-Whitney nonparametric test (STATA SE 8.0; StataCorp, College Station, Tex) was used to compare overall quality, image noise, and streak artifacts in each examination performed with combined modulation at different noise indexes or with the fixedtube current technique.

\section{Results}

There was no significant difference in transclavicular distance or scan coverage length among the 3 groups, indicating that subjects were similar in body size and scan volume.

There was a substantially significant difference in radiation dose among the 3 groups, with a DLP of $1179 \pm 109$ $(\mathrm{mGy}-\mathrm{cm})$ for fixed $\mathrm{mA}, 925 \pm 116$ for ATCM with a noise index of 11.4, and $729 \pm 148$ for ATCM with a noise index of 20.2 (Table 1). Compared with fixed-tube current, an overall reduction of $22 \%$ in DLP was achieved with the use of ATCM with a noise index of $11.4\left(P<.001 \times 10^{-7}\right)$ and an overall reduction of $38 \%$ in DLP with ATCM with a noise index of $20.2\left(P<.001 \times 10^{-11}\right)$. There was also a $21 \%$ reduction in DLP with use of a noise index of 20.2 compared with a noise 


\begin{tabular}{lcccc}
\hline \multicolumn{1}{l}{ Table 1: Summary of transclavicular distance, scan length, dose-length product (DLP), and CT dose index (CTDI) } \\
\hline Measurement & Transclavicular Distance (cm) & Scan Length (cm) & DLP (mGy-cm) & CTDI (mGy) \\
\hline Fixed mA & $20.1 \pm 1.3$ & $30.1 \pm 2.5$ & $1179 \pm 109$ & $34.7 \pm 2.2$ \\
NI 11.4 & $20.1 \pm 1.5$ & $30.1 \pm 2.7$ & $925 \pm 116$ & $27.8 \pm 3.5$ \\
NI 20.2 & $19.8 \pm 1.5$ & $29.4 \pm 4.4$ & $729 \pm 148$ & $23.0 \pm 3.5$ \\
\hline
\end{tabular}

Note:- NI indicates noise index; $\mathrm{mA}$, tube current-time product.

Table 2: $\boldsymbol{P}$ values of Student $\boldsymbol{t}$ test between two different imaging techniques

\begin{tabular}{lcccc}
\hline Measurement & $\begin{array}{c}\text { Transclavicular } \\
\text { Distance }\end{array}$ & $\begin{array}{c}\text { Scan } \\
\text { Length }\end{array}$ & DLP & CTDI \\
\hline Fixed mA vs NI 11.4 & 0.46 & 0.43 & $<0.008 \times 10^{-7}$ & $<0.008 \times 10^{-6}$ \\
NI 11.4 vs NI 20.2 & 0.92 & 0.89 & $<0.01 \times 10^{-3}$ & $<0.0001$ \\
Fixed mA vs NI 20.2 & 0.43 & 0.42 & $<0.007 \times 10^{-11}$ & $<0.004 \times 10^{-12}$ \\
\hline
\end{tabular}

Note:- NI indicates noise index; DLP, dose-length product; CTDI, CT dose index; mA, tube current-time product.

Image Quality, Noise, and Artifact

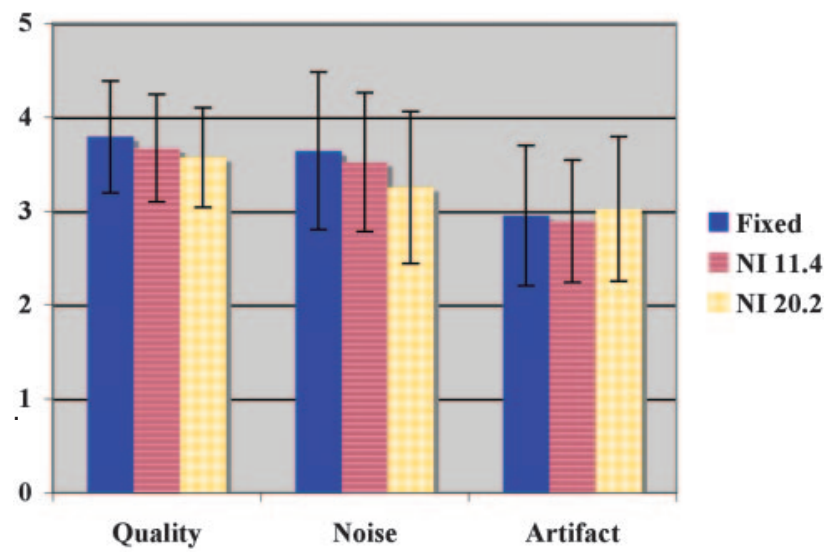

Fig 1. Pooled scores from 2 blinded readers regarding image quality, noise, and artifacts. There is minimal decrease in image quality, with slight increase in noise in ATCM techniques compared with the fixed $\mathrm{mA}$ technique. The magnitude of the difference is small (0.22) on a 5-point scale and does not seem to be clinically significant

index of $11.4\left(P<.01 \times 10^{-3}\right)$ (Table 2$)$. In a similar fashion, there was $20 \%$ of reduction in $\mathrm{CTDI}_{\mathrm{vol}}$ between fixed $\mathrm{mA}$ and a noise index of 11.4 and $34 \%$ reduction of $\mathrm{CTDI}_{\mathrm{vol}}$ between fixed $\mathrm{mA}$ and noise index 20.2 techniques $\left(P<.008 \times 10^{-6}\right.$, $P<.004 \times 10^{-12}$, respectively).

The pooled scores from the 2 readers regarding image quality, noise, and streak artifact (mean \pm SD) for images obtained with a fixed-tube current, ATCM with a noise index of 11.4 and 20.2 are summarized in Fig 1. There was only minimal decrease in image quality, with increased noise between the fixed-tube current and ATCM with a noise index of 11.4 and between a noise index of 11.4 and 20.2 (Fig 1). There was a statistically significant decrease in image quality and an increase in image noise between the fixed-tube current and ATCM noise index of $20.2(P=.029$ and $P=.017$, respectively), indicating that the ATCM technique delivers lower image quality and higher noise compared with the fixed $\mathrm{mA}$ technique. However, the magnitude of the difference was felt to be small, as the average score of fixed $\mathrm{mA}$ was $3.79( \pm 0.59)$ and that of noise index 20.2 was 3.57 ( \pm 0.53 ), where a score of 3 indicates an "acceptable" image quality (Fig 2-4). No significant difference in streak artifact was observed among the different imaging techniques.

\section{Discussion}

In this preliminary analysis, we found a significant reduction in radiation dose with the use of ATCM compared with a fixed $\mathrm{mA}$ technique for volume neck CT. Although there was a small difference in pooled subjective scores on image quality and noise, the magnitude of the difference was small. CT examination of the neck with use of the ATCM technique with a noise of 11.4 and 20.2 provides image quality that is diagnostically acceptable. Therefore, in keeping with the ALARA concept, we endorse the use of ATCM with a noise index of 20.2 for MDCT of the neck.

ATCM was developed to minimize subjective estimation and selection of tube current required to obtain desired image quality. ${ }^{4,5}$ Shortly after installation of 64 -section MDCT at our institution, the fixed $\mathrm{mA}$ technique was used exclusively. The tube current in this setting was manually selected by CT technologists by subjective estimation of patients' body size, often resulting in inconsistent image quality. The neck was an ideal body part in which to use combination ATCM because of its widely varying shape and attenuation within the subject and the presence of highly radiosensitive organs (ie, thyroid and lens) in CT examinations of the neck. To the best of our knowledge, there is no previously published data evaluating the use of combination ATCM (z-axis and angular modulation) in neck CT. These findings are in agreement with previously published reports regarding CT of the chest, abdomen, and pelvis with use of ATCM. ${ }^{6-11}$

In August 2001, the Society of Pediatric Radiology organized a multidisciplinary ALARA conference in that the consensus was a statistically significant, albeit small, individual risk for excess cancer in children from ionizing doses of radiation used in helical CT. On the basis of new and more extensive data, the Committee on the Biologic Effects of Ionizing Radiation VII supported a "linear-no-threshold" model, which states that the risk for cancer in humans proceeds in a linear fashion at lower doses without a "safe" threshold, and that even the smallest dose has the potential to cause a small increase in risk to humans. ${ }^{13}$

Although reduction in radiation dose is an important exercise, maintaining high quality of a diagnostic imaging study is also essential to provide an accurate and definitive diagnosis. We must keep a fine balance between image quality and radiation dose. One could argue that radiation exposure from a diagnostic CT imaging scan is relatively small for patients with head and neck cancer who undergo therapeutic irradiation, 

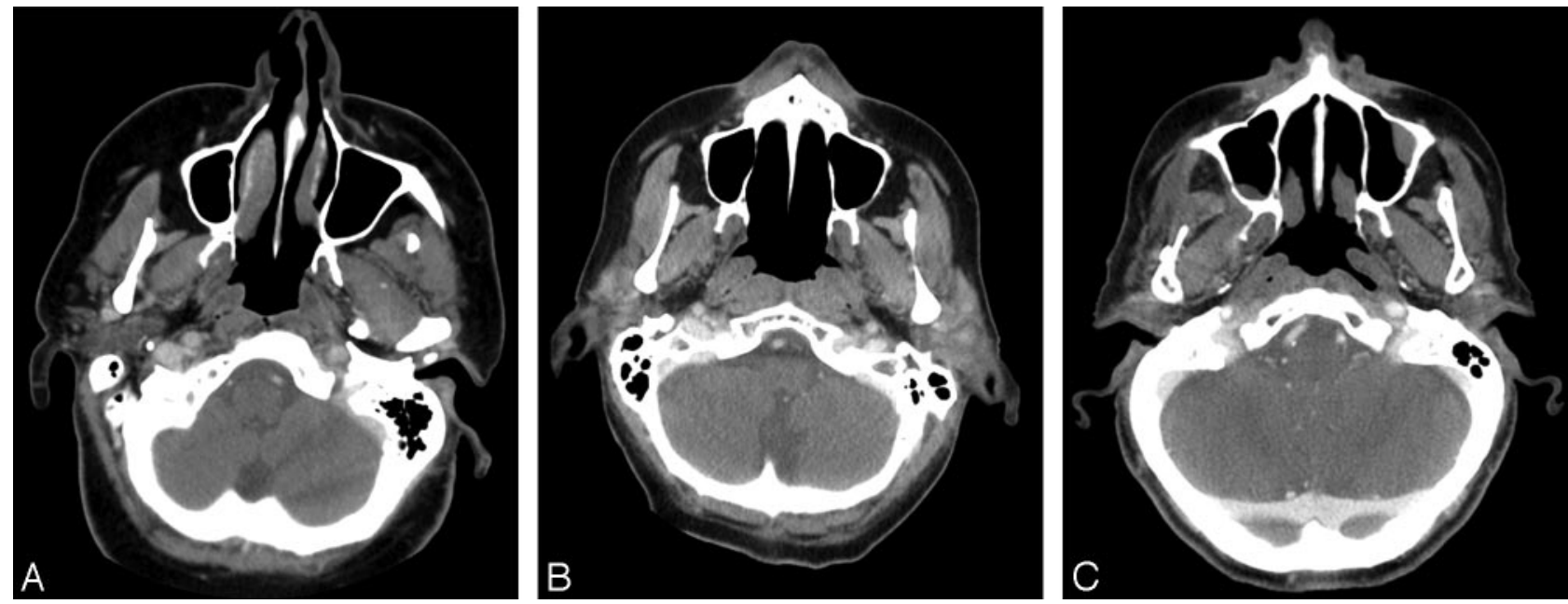

Fig 2. CT images through the posterior fossa with use of the fixed-tube current technique (A), ATCM with noise index of $11.4(B)$, and ATCM with noise index of $20.2(C)$. There is no perceivable difference in overall image quality among the 3 different techniques. All images are of diagnostic quality.
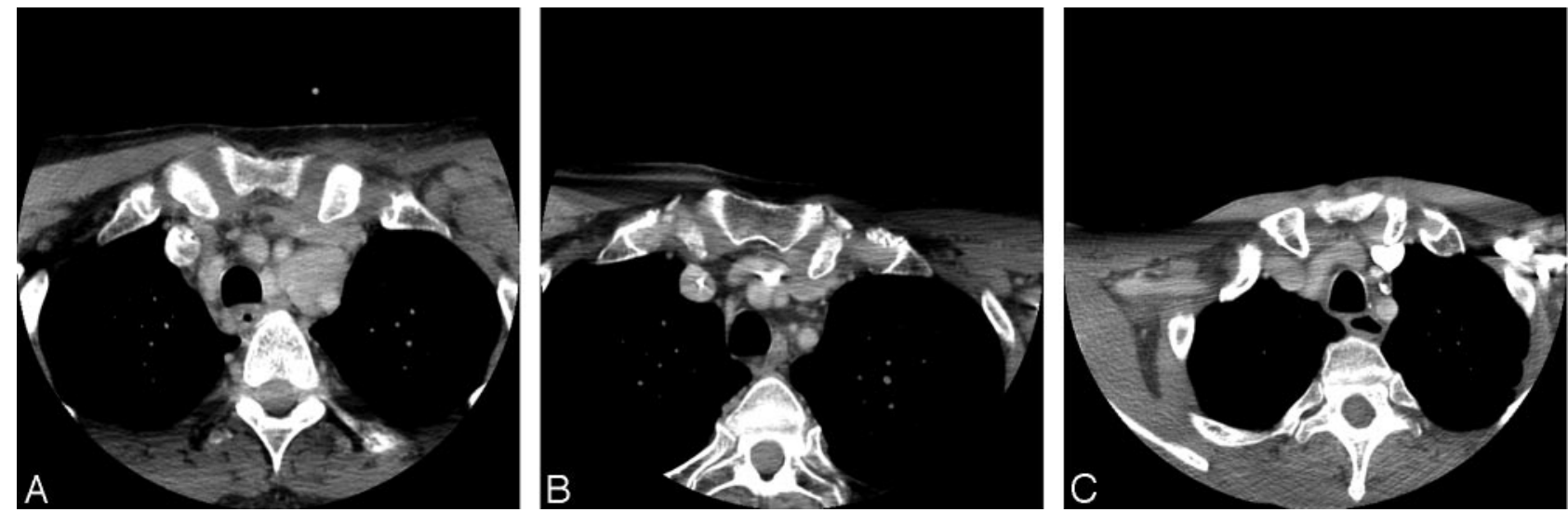

Fig 3. CT images through thoracic inlet with use of fixed-tube current technique (A), ATCM with noise index of $11.4(B)$, and ATCM with noise index of 20.2 (C). There is no perceivable difference in image quality and noise level. No difference in the degree of streak artifact is noted.
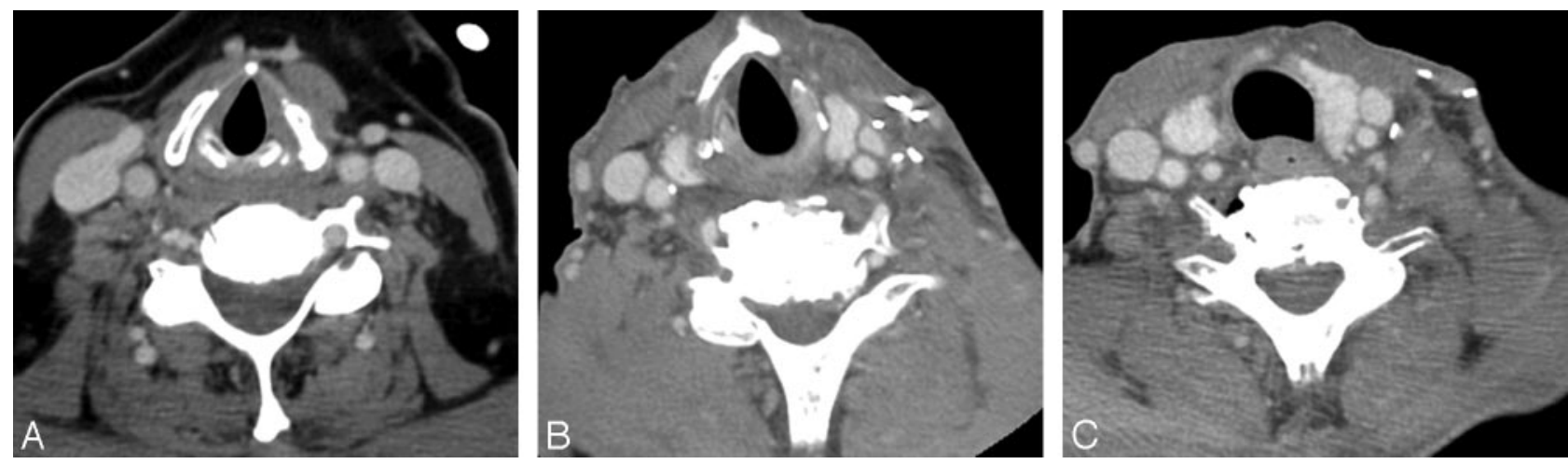

Fig 4. CT images through the neck with use of the fixed-tube current technique (A), ATCM with noise index of $11.4(B)$, and ATCM with noise index of 20.2 (C). There is no perceivable difference in overall image quality. All images are of diagnostic quality.

compared with young patients who are suspect for infectious or inflammatory disease. Imaging techniques such as noise index can be potentially tailored to individual patient needs and risks in the future.

Limitations of this study were the small sample size and the retrospective collection of study materials, which might have introduced selection bias. In addition, further investigation needs to be performed to better define an "accept- able" image quality for head and neck radiologists. Blinded assessment of image quality may not be the most robust measures of image quality. Moreover, accuracy or conspicuity of abnormality was not addressed in this study. The ultimate threshold between acceptable and unacceptable imaging quality might be defined as a noise index high enough to start missing an abnormal lesion. This is not practical to investigate in the clinical setting. It is highly 
likely that acceptable image quality could vary depending on individual radiologists.

Patient safety and quality of care have been discussed extensively in health care organizations including the Centers for Medicare \& Medicaid. One of the important quality metrics in diagnostic radiology is radiation dose. ${ }^{14}$ Judicious monitoring of radiation dose to patients is highly essential to ensure patient safety, and such efforts have to be balanced with diagnostic quality of imaging examinations.

\section{Conclusions}

ATCM technique substantially reduced radiation dose to patients without significantly compromising image quality of 64-section MDCT of the neck. The neck is an ideal body part to apply ATCM because of its widely varying shape and attenuation within a subject and the presence of highly radiosensitive organs (ie, thyroid and lens). With the ALARA concept, institutional effort of balancing image quality and radiation dose to patients is essential for providing high quality of clinical care and patient safety.

\section{References}

1. Mettler FA Jr, Wiest PW, Locken JA, Kelsey CA. CT scanning: patterns of use and dose. J Radiol Prot 2000;20:353-59
2. Rehani MM. Effects of low level ionising radiations. Indian J Biochem Biophys 1999;36:365-73

3. Lee CI, Haims AH, Monico EP, et al. Diagnostic CT scans: assessment of patient, physician, and radiologist awareness of radiation dose and possible risks. Radiology 2004;231:393-98

4. Kalra MK, Maher MM, Toth TL, et al. Strategies for CT radiation dose optimization. Radiology 2004;230:619-28

5. Kalra MK, Maher MM, Toth TL, et al. Techniques and applications of automatic tube current modulation for CT. Radiology 2004;233:649-57

6. Kalra MK, Maher MM, Toth TL, et al. Comparison of $\mathbf{z}$-axis automatic tube current modulation technique with fixed tube current CT scanning of abdomen and pelvis. Radiology 2004;232:347-53

7. Kalra MK, Maher MM, D'Souza RV, et al. Detection of urinary tract stones at low-radiation-dose CT with z-axis automatic tube current modulation: phantom and clinical studies. Radiology 2005;235:523-29

8. Campbell J, Kalra MK, Rizzo S, et al. Scanning beyond anatomic limits of the thorax in chest CT: findings, radiation dose, and automatic tube current modulation. AJR Am J Roentgenol 2005;185:1525-30

9. Kalra MK, Rizzo S, Maher MM, et al. Chest CT performed with z-axis modulation: scanning protocol and radiation dose. Radiology 2005;237:303-08

10. Mulkens TH, Bellinck P, Baeyaert M, et al. Use of an automatic exposure control mechanism for dose optimization in multi-detector row CT examinations: clinical evaluation. Radiology 2005;237:213-23

11. Rizzo S, Kalra M, Schmidt B, et al. Comparison of angular and combined automatic tube current modulation techniques with constant tube current CT of the abdomen and pelvis. AJR Am J Roentgenol 2006;186:673-79

12. Morin RL, Gerber TC, McCollough CH. Radiation dose in computed tomography of the heart. Circulation 2003;107:917-22

13. Tubiana M, Aurengo A, Averbeck D, et al. The debate on the use of linear no threshold for assessing the effects of low doses. J Radiol Prot 2006;26:317-24

14. Moser JW, Wilcox PA, Bjork SS, et al. Pay for performance in radiology: ACR white paper. J Am Coll Radiol 2006;3:650-64 